\title{
Electric Field Determination in DC Polymeric Power Cable in the Presence of Space Charge and Temperature Gradient under dc Conditions
}

\author{
W. Choo and G. Chen \\ University of Southampton, School of Electronics and Computer Science, Southampton SO17 1BJ, United Kingdom \\ *Email: ctc05r@ecs.soton.ac.uk
}

\begin{abstract}
In direct current (dc) power cable the electric field is affected by conductivity of the material, which is a function of both temperature and electric field. Loading in high voltage direct current (HVDC) cable results in a temperature gradient across the insulation and hence a radial distribution of the insulation conductivity. A direct consequence is the accumulation of space charge within the insulation bulk, which modifies the electric field across the insulation. The coupled problems due to electric field and temperature inflict difficulties to identify the electric field distribution in HVDC cables, which therefore poses threat to the reliability in operation of dc power cables. Space charge in a commercial $11 \mathrm{kV}$ ac XLPE power cable has been measured under a voltage application of $80 \mathrm{kV}$ dc. Space charge distributions were obtained across the insulation of the cable by means of a modified pulsed electroacoustic (PEA) system. In this paper, COMSOL Multiphysics software package is also used to accurately determine the electric field distribution in the dc power cable by considering the influences of both the effects of temperature and electric field on the conductivity of the insulating material.
\end{abstract}

Index Terms--Space charge, electric field, XLPE, PEA, conductivity, temperature gradient, dc power cable.

\section{INTRODUCTION}

$\mathrm{F}_{\mathrm{v}}^{\mathrm{o}}$ or the past few years, polymeric insulations for high voltage direct current (HVDC) applications have drawn a high level of interest. Currently, most HVDC installations used around the world uses traditional oil-impregnated paper insulation and oil-filled-type cables [1]. Polymeric materials like XLPE have long been the preferred insulation material and are gradually replacing traditional oil-impregnated paper insulation due to their low material and processing costs as well as their excellent high dielectric strength and electrical resistivity in combination with excellent physical properties such as resistance to cracking and moisture penetration [2]. Presently, cross-linked polyethylene (XLPE) insulation has been used, very successfully, for underground transmission and distribution of power, using high voltage alternating current (HVAC) transmission. DC power cables are also typically more cost-effective as compared to its counterpart of $\mathrm{AC}$ power cable due to the low conductor loss and thinner insulation thickness for the same power rating.

The main reason that prevents the use of polymers as insulation for high voltage direct current cables for the electricity generation and supply industries lies to the degradation of polymer insulation under high electric stress. This phenomenon is associated with the formation of space charge. In fact, space charge accumulation in polymeric materials under dc regime poses major scientific and technological problems.

However, the influence of space charge on the electric field distribution in cable so far has been obtained without the considering effect of both temperature and electric field on conductivity. This will result in some error in electric field distribution as it is well known fact that electric field under do conditions is determined by conductivity.

In general, the influence of space charge field on the conductivity and subsequently electric field distribution within the polymeric material has been considered. The space charge distributions in the polymeric materials are measured using the pulsed electro-acoustic (PEA) technique. A numerical simulation using COMSOL Multiphysics software package is also used in this project, which allows us to provide a clear overview on the underlying theoretical principles and phenomenon of space charge through the determination of results obtained from the polymeric material.

\section{GENERAL EXPRESSION FOR DC CONDUCTIVITY}

Electric field is resistively graded under the application of direct current $(\mathrm{dc})$. The influence effect of both temperature and electric field show a strong relationship with dc conductivity [1]. With load being considered, major effect on field grading could be seen when there is a variation in conductivity with temperature in the insulation. It could be view that for a small temperature gradient, the electric field distribution is almost uniform. The stress is inverted when the temperature gradient is increased to an appreciably degree. Conversely, for the field-dependence of the conductivity, it is in general considered to be a second order effect as the effect is comparatively small.

The derivation of conductivity could be done based on the hopping theory model of conduction in dielectric [3] and is expressed as

$$
\sigma(T, E)=A \exp \left(-\frac{\varphi q}{k T}\right) \frac{\sinh (B|E|)}{|E|}
$$


where $\mathrm{A}$ and $\mathrm{B}$ are constants, $\varphi$ the thermal activation energy in $\mathrm{eV}, \mathrm{q}$ the elementary charge, $\mathrm{T}$ the temperature in $\mathrm{K}$, $\mathrm{E}$ the electric field in $\mathrm{V} / \mathrm{m}$ and $\mathrm{k}$ the Bolzmann constant.

The above equation is applied in COMSOL to compute the electric field distribution in cables in the absence of space charge. It was examined that constants A, B and the thermal activation energy do varies depending on the materials and the conditions of it.

$$
\begin{aligned}
& \sigma_{\text {Good }}(T, E)=3.2781 \exp \left(-\frac{0.56 q}{k T}\right) \frac{\sinh \left(2.7756 \times 10^{-7}|E|\right)}{|E|} \\
& \sigma_{\text {Bad }}(T, E)=3.6782 \times 10^{7} \exp \left(-\frac{0.98 q}{k T}\right) \frac{\sinh \left(.086 \times 10^{-7}|E|\right)}{|E|}
\end{aligned}
$$

Boggs et al [4] have demonstrated the parameters classification and classified them between two groups, good and bad dielectric respectively for XLPE cable as represented in equations (2) and (3) respectively.

\section{EXPERIMENTAL DETAILS}

The sample tested is a commercial $11 \mathrm{kV}$ ac XLPE insulated power cable (inner radii $=8.75 \mathrm{~mm}$; outer radii $=$ $11.55 \mathrm{~mm}$; insulation thickness $=2.8 \mathrm{~mm}$ ). In order to understand the influence of crosslinking by-products (residues) in the space charge formation, the cable did not undergo the degassing process therefore contains crosslinking by-products.

The cable was then stressed at $80 \mathrm{kV}$ for 20 hours and space charge distributions were subsequently measured immediately after the removal of the applied voltage as shown in Figure 1. A modified PEA system [5] where a flat ground electrode was utilised instead of curved ground electrode from a conventional cable PEA system [6].

As illustrated in Figure 1, dominating heterocharge formation is obtained in the XLPE insulation. Due to after crosslinking process, there will be formation of by-products such as methane, cumylalcohol, acetophenone etc. in the XLPE cable. Without degassing process these by-products may be present in the material for a long period of time and undergo ionisation process under high electric field. Ionised species will move towards the opposite electrodes to form heterocharge. As the extraction of charge at the counterelectrode may be slow and this mechanism might be a potential cause.

The charge distribution shape and magnitude changes with time. As stressing time increases, both injected negative and positive charges start to move further into the sample and getting closer to each other. Both negative and positive magnitude increase significantly with the duration of applied voltage [7]. Experiments by $\mathrm{M}$. Fu also revealed that the space charge formed after 20 hours of stressing is very stable [8], implicating that the charges are deeply trapped.

Once the space charge distribution $\rho(\mathrm{r})$ is obtained in the sample material, the electric field ESC(r) produced by space charge can be calculated based on Poisson's equation represented by

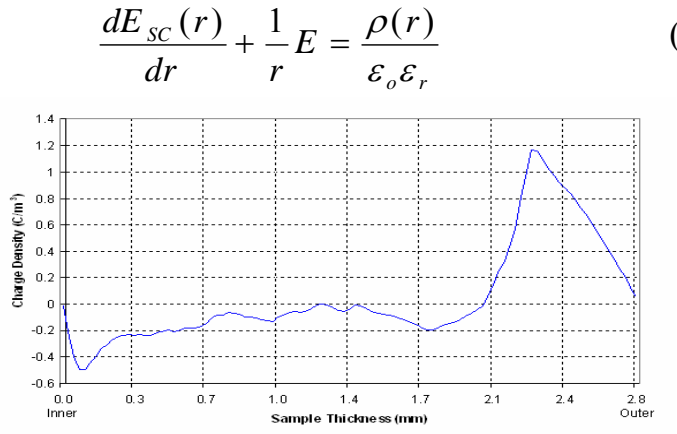

Figure 1: Space charge distribution across cable insulation.

where $\varepsilon 0$ is the permittivity of vacuum and $\varepsilon r$ the relative permittivity of the material. Generally, it is believed that the field-dependence of the conductivity is relatively small. Therefore, the total field in the published work is obtained by summing the applied field and this field due to space charge and is expressed as

$$
E_{\text {Total }}=E_{\text {Applied }}+E_{S C}
$$

\section{SIMULATION RESULTS AND DISCUSSIONS}

In the present simulation, we have utilised the parameters from good and bad material as stated in equation (2) and (3) respectively to obtain an accurate electric field distribution through considering the influence of space charge field and conductivity [4].

Three steps have been adopted as follows

(i) Calculation of the electric field due to space charge based on Poisson's equation.

(ii) The electric field is being calculated based on the conductivity equation to determine the electric field arisen from the applied voltage.

(iii) Finally adding both the two fields together to form the total electric field as indicated in equation (5).

Applying the classification of good parameters, the simulated results with an application of $80 \mathrm{kV}$ is applied to the cable where field-dependent and no occurrence of temperature gradient conductivity is being considered. The conductivity will be slightly linear as the conductivity decrease gradually from the inner to the outer electrode when space charge is ignored [9].

With consideration of the space charge field, Figure $2 \mathrm{a}$ clearly shows that with the accumulated space charge, the voltage profile deviate to non-linearity at the region adjacent to the interface. Conductivity of the sample material is no longer linear due to the consideration of space charge field. As shown in Figure $2 \mathrm{~b}$, maximum conductivity of $1.08 \times 10-13$ $\mathrm{S} / \mathrm{m}$ occurs at the outer interface. The electric field distribution due to applied voltage is varied greatly as shown in Figure 2c, where the minimum occurring at the outer interface and the maximum of $34 \mathrm{kV} / \mathrm{mm}$ occurring between the middle of the sample and the outer interface.

With the consideration of temperature gradient between the inner and outer interface within the cable, Figure $3 b$ clearly shows that there is an increase in conductivity distribution. In 
Figure 3c, when temperature gradient increases from $30 \mathrm{~K}$ to $50 \mathrm{~K}$, it shows that electric field distribution due to applied voltage actually decrease at the inner region and tends to increase at the middle of the sample to the outer interface of the insulation.

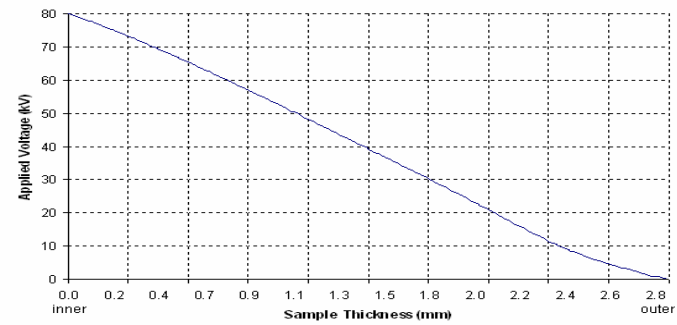

(a) Voltage distribution

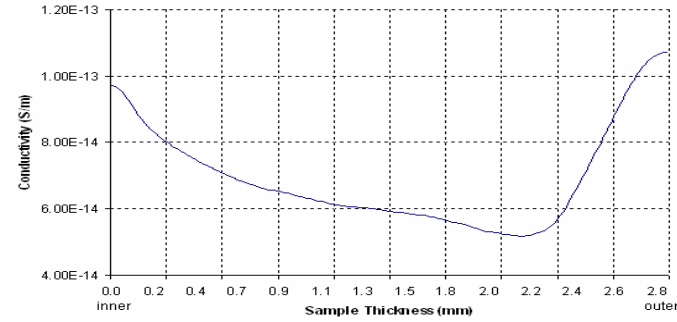

(b) Conductivity distribution

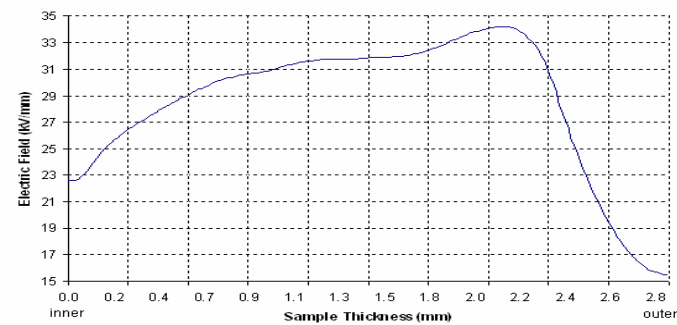

(c) Electric field distribution due to applied voltage

Figure 2: Simulation results considering the field-dependent conductivity when $0 \mathrm{~K}$ temperature gradient $T_{\text {inner }}=295 \mathrm{~K}$ and $T_{\text {outer }}=295 \mathrm{~K}$ with the presence of heterocharge.

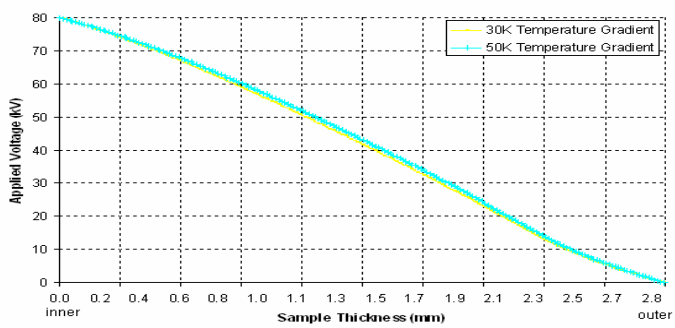

(a) Voltage distribution

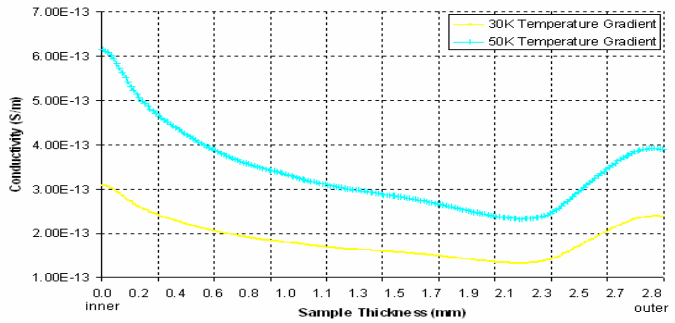

(b) Conductivity distribution

The calculated total electric field distribution in the material is illustrated in Figure 4. When the conductor of cable is heated, the maximum stress occurs at the outer region adjacent to the screen of the cable. With the increment in temperature gradient, the stress at the inner region tends to decrease more and the stress at the screen of the cable increases more.

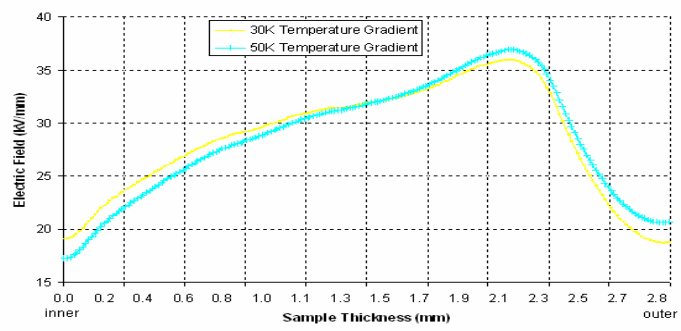

(c) Electric field distribution due to applied voltage

Figure 3: Simulation results considering both field and temperature gradient dependent on conductivity $\mathrm{T}_{\text {outer }}=295 \mathrm{~K}$.

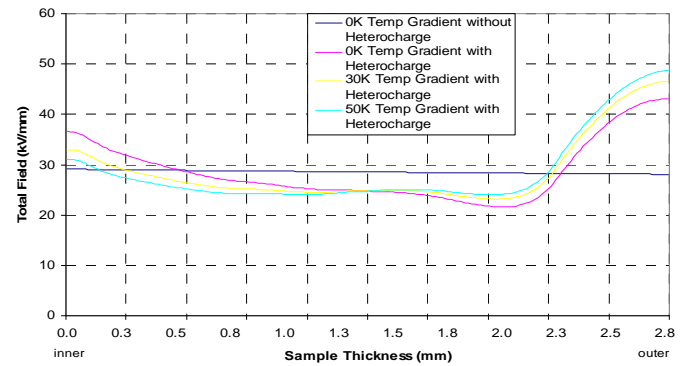

Figure 4: Total electric field distribution in cable with $T_{\text {outer }}=295 \mathrm{~K}$ based on Good Parameter.

The simulation results obtained above are all based on parameters from a good dielectric material. Conductivity equation (2) indicates that it has stronger field dependence, which means when there is a small variation in electric field, the conductivity changes significantly. Therefore, resulting a stronger modification of the electric field. Conversely, if we choose the parameters for a bad dielectric material by using equation (3), one should expect there will be less field modification.

As illustrated in both Figures 4 and 5, comparisons were made between the electric field distributions of good and bad parameters insulation at 20 hours. Our simulation results suggest there is no significant difference when no temperature gradient is considered. This insignificance may be related to the final value of conductivity of the material. The conductivity distribution for both good and bad materials is determined by space charge field which is the same. Nevertheless, when temperature gradient is considered, it is shown that there is a significant difference in results through the adopting between good and bad dielectric parameters. As illustrated on the figures with comparison between good and bad dielectric parameters with temperature gradients being considered, it shows that there is not much variation in terms of stress when good parameters are adopted as compared with a significant variation when bad parameters are adopted.

The difference in the total electric field in terms of temperature gradient for $30 \mathrm{~K}$ and $50 \mathrm{~K}$ are $20 \%$ and $28 \%$ respectively between good and bad dielectric materials. From 
the simulation results we obtained, it can be suggested that bad dielectric parameters as indicated in equation (3) shows stronger temperature dependence.

Previously, the numerical model was also applied to a cable with an accumulation of homocharge in the XLPE dielectric material due to charge injection from the two electrodes [9].

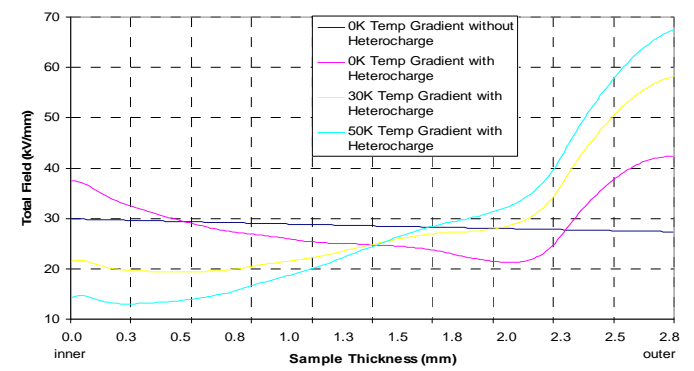

Figure 5: Total electric field distribution in cable with $T_{\text {outer }}=295 \mathrm{~K}$ based on Bad Parameter.

\section{CONCLUSION}

We have measured space charge across the insulation in a dc power cable after an application of $80 \mathrm{kV}$ for 20 hours using the PEA technique. In this experiment, heterocharge is accumulated in the XLPE dielectric material of the cable due to the influence of crosslinking by-products (residues) in the space charge formation as the cable did not undergo the degassing process therefore contains crosslinking by-products.

COMSOL Multiphysics software package is used to accurately determine the electric field distribution in the dc power cable by considering the influences of both the effects of temperature and electric field on the conductivity of the insulating material. The results indicate that when a cable is heated, the maximum stress occurs at the outer region adjacent to the screen of the cable and the value of this stress increases with the increase value of temperature drop across the dielectric material. Comparisons were also made between good and bad dielectric parameters with temperature gradients being considered, it shows that there is not much variation in terms of stress when good parameters are adopted as compared with the bad parameters adopted where significant variation were observed.

Bulk space charge accumulation caused by non-uniform conductivity due to temperature gradient and electric field is therefore essential to both cable designers as well as the users.

\section{REFERENCES}

[1] B. M. Weedy and D. Chu, "HVDC extruded cables - Parameters for determination of stresses, "IEEE Trans PAS. Vol. 103, pp. 662-667, 1984.

[2] L. A. Dissado and J. H. Fothergill, Electrical degradation and breakdown in polymers, London, Peter Peregrinus Ltd, 1992.

[3] A. R. Blythe and D. Bloor, Electrical properties of Polymers, 2nd Edition, Cambridge University Press, 2005

[4] S. Boggs, D. Damon, J. Hjerrild, J. Holbol and M. Henriksen, "Effect of Insulation Properties on the Field Grading of Solid Dielectric DC Cable.”, IEEE TPD, Vol. 16, No.4, pp.456-461, 2001.
[5] M. Fu and G. Chen, "Space charge measurement in polymer insulated power cables using flat ground electrode PEA system," IEE Proc.Science, Measurement \& Technology, Vol. 150, pp. 89-96, 2003.

[6] N. Hozumi, H. Suzuki, T. Okamoto, K. Watanabe and A. Watanabe, "Direct observation of time-dependent space charge profiles in XLPE cable under high electric fields", IEEE Trans. Dielectr. Electr. Insul., Vol. 1, pp. 1068 - 1076, 1994.

[7] M. Fu, G. Chen, L. A. Dissado and J. C. Fothergill, "Influence of Thermal Treatment and Residues on Space Charge Accumulation in XLPE for DC Power Cable Application", IEEE Trans. on Dielectr. Electr. Insul., Vol. 14, No.1; Feb 2007.

[8] M. Fu, "Space charge measurement in polymer insulated power cables using the PEA method", PhD thesis, University of Southampton, UK, 2002.

[9] W. Choo and G. Chen, "Electric Field Determination in DC Polymeric Power Cable in the Presence of Space Charge", 2007 Annual Report Conference on Electrical Insulation and Dielectric Phenomena, pp. 489492, 2007. 